American Journal of Theoretical and Applied Statistics
2021; 10(1): $72-88$
http://www.sciencepublishinggroup.com/j/ajtas
doi: 10.11648 /j.ajtas.20211001.18
ISSN: $2326-8999$ (Print); ISSN: $2326-9006$ (Online)

\title{
Studying Changes on Stock Market Transactions Using Different Techniques for Multivariate Time Series
}

\author{
Ahmed Mohamed Mohamed Elsayed \\ Department of Basic Science, Al-Obour High Institute for Management \& Informatics, Obour City, Egypt \\ Email address: \\ atabl18@yahoo.com, atabl@oi.edu.eg \\ To cite this article: \\ Ahmed Mohamed Mohamed Elsayed. Studying Changes on Stock Market Transactions Using Different Techniques for Multivariate Time \\ Series. American Journal of Theoretical and Applied Statistics. Vol. 10, No. 1, 2021, pp. 72-88. doi: 10.11648/j.ajtas.20211001.18
}

Received: February 2, 2021; Accepted: February 14, 2021; Published: February 26, 2021

\begin{abstract}
There are many studies dealt with univariate time series data, but the analysis of multivariate time series are rarely discussed. This article discusses the theoretical and numerical aspects of different techniques that analyze the multivariate time series data. These techniques are ANN, ARIMA, GLM and VARS models. All techniques are used to analyze the data that obtained from Egypt Stock Exchange Market. R program with many packages are used. These packages are the "neuralnet, nnet, forecast, MTS and vars". The process of measuring the accuracy of forecasting are investigated using the measures ME, ACF, MAE, MPE, RMSE, MASE, and MAPE. This is done for seasonal and non-seasonal time series data. Best ARIMA model with minimum error is constructed and tested. The lags order of the model are identified. Granger test for causality indicated that Exchange rate is useful for forecasting another time series. Also, the Instant test indicated that there is instantaneous causality between Exchange rate and other time series. For non-seasonal data, the NNAR() model is equivalent to ARIMA() model. Also, for seasonal data, the NNAR(p,P,0)[m] model is equivalent to an $\operatorname{ARIMA}(\mathrm{p}, 0,0)(\mathrm{P}, 0,0)[\mathrm{m}]$ model. For these data, we concluded that the ANN and GLMs of fitting multivariate seasonal time series is better than multivariate non-seasonal time series. The transactions of Finance, Household and Chemicals sectors are significant for Exchange rate in non-seasonal time series case. The forecasts that based on stationary time series data are more smooth and accurate. VARS model is more accurate rather than VAR model for ARIMA $(0,0,1)$. Forecasts of VAR values are predicted over short horizon, because the prediction over long horizon becomes unreliable or uniform.
\end{abstract}

Keywords: ANN, GLM, ARIMA, VARS, Backpropagation, RMSE, Causality Test, Instant Test

\section{Introduction}

Time series analysis is one of the most important processes that many companies and even many countries need. These companies or countries need to forecast the behavior of some phenomenon in the future. Not only for univariate time series analysis but also for multivariate time series analysis. Artificial neural networks (ANN) have become one of the most important methods of artificial intelligence in the processes of forecasting, and given that many recent articles do not deal much with the processes of multivariate analysis, whether by the autoregressive integrated moving average (ARIMA) models or ANNs models. We will combine both methods to forecast multivariate time series about applications are based on real data using some of $\mathrm{R}$ program packages. In addition to these two methods, we will use both the vector autoregressive models (VARS) and the generalized linear models (GLMs) for multivariate time series analysis, and then try to find which one of these methods is better.

The predictors form the ANN bottom layer, and the forecasts form the top layer. Intermediate layers containing hidden neurons. The simplest networks contain no hidden layers, this is equivalent to linear regressions. The coefficients attached to these predictors called weights. The weights are selected in the $\mathrm{NN}$ framework using a learning algorithm that minimizes the costs. The number of nodes in each hidden layer must be specified. We can consider ANN as a nonlinear statistical data. Complex relations between inputs and outputs are happened. ANNs have been applied to numerous applications in many field including pharmaceutical research, engineering and medicinal chemistry. ANNs were used in drug discovery. It allows the estimation of some non-linear models without need to define an accurate functional. There are many ancient and recent researches and articles have 
presented the comparisons between the ARIMAs and the ANNs, in the process of forecasting the univariate time series.

Intrator and Intrator [1] used $\mathrm{NN}$ for interpretation of nonlinear models. Zhang et al. [2] introduced the forecasting with ANNs. A comparison between $\mathrm{NN}$ and Box-Jenkins forecasting techniques with application to real data devoted by Al-Shawadfi [3]. Hothorn et al. [4] have designed and analysis of benchmark experiments. LiHong et al. [5] devoted the NNs based drug discovery approach and its application for designing aldose reductase inhibitors. Zou and Zhou [6] presented QSAR study of oxazolidinone antibacterial agents using ANNs. Eugster et al. [7] introduced exploratory and inferential analysis of benchmark experiments. Kose [8] presented modelling of color perception of different age groups using ANNs. Al-Shawadfi and Hagag [9] have suggested that the ANN approach may provide a superior alternative to the Box-Jenkins forecasting approach for developing forecasting models in situations that do not require modeling of the internal structure of the series. The numerical results showed that the approach has a good performance for the forecasting of ARMAX models. Rostampour et al. [10] used an ANN for prediction of apple bruise damage. Doreswamy and Chanabasayya [11] presented a performance analysis of NN models for oxazolines and oxazoles derivatives descriptor dataset. Hanjouri and Qamar [12] have devoted two methods for analyzing and forecasting time series data. The two methods aimed to compare Box and Jenkins models, and the ANN to forecast global sugar prices. The analysis of sugar price data reversed the superiority of the ARIMA time series model and gave more accurate predictions than ANNs.

This paper discusses the theoretical aspects of different techniques and models that analysis time series data and will concentrate on the multivariate time series data. These techniques are ANN, ARIMA, GLM and VARS models. All models are used to analyze the multivariate time series data that obtained from Egypt Stock Exchange Market. The R program with many packages and functions are used.

This paper can be organized as follow: Section 2 presents some materials, algorithms and models that deal with multivariate ordinary, time series, seasonal data. Section 3 presents the numeric analysis section divided into some subsections devoted the features of dataset, and the results of analysis dataset using the previous methods. Section 4 presents the discussion of obtained results for all models. Section 5 presents the conclusions of this article

\section{Materials, Algorithms and Models}

In this section, we will refer to some materials, algorithms and models that are used in this paper:

\subsection{ARIM Models}

ARIMA model is a generalization of ARMA model. Both of them fitted to time series data either to better understand or forecast. ARIMA models are applied where there non-stationary case. Non-seasonal ARIMA models denoted
$\operatorname{ARIMA}(\mathrm{p}, \mathrm{d}, \mathrm{q})$, where $\mathrm{p}$ is an order of the autoregressive model, $\mathrm{d}$ is the degree of differencing, and $\mathrm{q}$ is the order of the MA model. An order $\mathrm{p}$ and $\mathrm{q}$ can be determined using the sample autocorrelation function (ACF), partial autocorrelation function (PACF). Other alternative methods like AIC, BIC, etc. are used to determine an order of a non-seasonal ARIMA model. Seasonal ARIMA models are denoted $\operatorname{ARIMA}(\mathrm{p}, \mathrm{d}, \mathrm{q})(\mathrm{P}, \mathrm{D}, \mathrm{Q})[\mathrm{m}]$, where $\mathrm{m}$ refers to the number of periods in each season. P,D,Q refer to the autoregressive, differencing, and moving average terms for the seasonal part of the ARIMA model. ARIMA(1,0,0)represents AR(1), ARIMA represents $\mathrm{I}(1)$, and $\operatorname{ARIMA}(0,0,1)$ represents MA(1). ARIMA models can be estimated according to Box-Jenkins technique. VARIMA model may be suitable if multiple time series are used then. Sometimes a seasonal effect on the model, SARIMA (Seasonal ARIMA) model is better to use. The non-stationary ARIMA model can be written as:

$$
Y_{t}=1-(1-L)^{d} X_{t}
$$

Where $t$ is an integer index, $L$ is the lag operator, and the $X_{t}$ are time series data, and $d$-dimensional multivariate time series.

While the stationary ARIMA cab be written as:

$$
\left(1-\sum_{i=1}^{p} \varphi_{i} L^{i}\right) Y_{t}=\left(1+\sum_{i=1}^{p} \theta_{i} L^{i}\right) \varepsilon_{t}
$$

Where $\varphi_{i}$ are the parameters of $\mathrm{AR}$ part, $\theta_{i}$ are the parameters of MA part, and $\varepsilon_{t}$ are error terms. The $\varepsilon_{t}$ are generally assumed to be "iid" variables from normal distribution with zero mean. Useful criterion is AIC:

$$
\mathrm{AIC}=-2 \log (L)+2(p+q+k)
$$

Where $L$ is the likelihood function, $p$ is the order of AR part and $q$ is the order of MA part, and $k$ represents the intercept of the ARIMA model.

$$
\mathrm{AICc}=\mathrm{AIC}+\frac{2(p+q+k)(p+q+k-1)}{T-p-q-k-1}
$$

$\mathrm{BIC}$ criterion is:

$$
\mathrm{BIC}=A I C+\left(\log _{e}(T)-2\right)(p+q+k)
$$

The aim is minimizing AIC, AICc or BIC values for a good model. The lower value, the better model. AIC tries to approximate models towards the reality. BIC attempts to find the perfect fit. BIC is useful method for selection models having more parameters. AICc is used to compare ARIMAs with same orders differencing. Also, the root of mean squared error (RMSE) can be used for comparing ARIMAs with different orders differencing. [13-17]

\subsection{Artificial Neural Networks (ANNs)}

ANN consists of Input layers, Hidden layers, and Output 
layers. A first layer of ANN receives the raw input, processes it, using Back-propagation algorithm, and passes the processed information to the hidden layers. The hidden layer passes the information to the last layer, which produces the output. ANN is characterized by an activation function. The activation function is often defined by the range $[-1,1]$ or $[0,1]$, and that function may be is linear or nonlinear, and can be in several shapes like:

(1). $g(x)=1-\frac{2}{1+\mathrm{e}^{2 x}}$.

(2). $g(x)=1+\frac{1}{1+\mathrm{e}^{-x}}$.

(3). $g(x)= \begin{cases}0, & \text { if } x<0, \\ 1, & \text { otherwise. }\end{cases}$

(4). $g(x)=\mathrm{e}^{\frac{x^{2}}{2}}$.

A perceptron (single layer ANN) receives multidimensional input and processes it using a weighted summation and an activation function. A major limitation of perceptron model is its inability to deal with non-linearity. A multilayered ANN overcomes this limitation and helps to solve non-linear problems. Figure 1 displays the simple ANN structure:

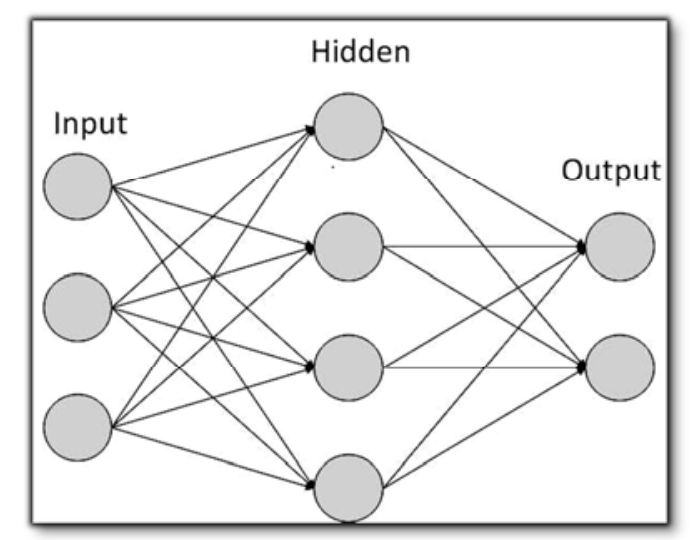

Figure 1. Artificial Intelligence-Simple ANN-Tutorials point

The most basic type of ANN is called Feed-forward, an information flow in only one direction. A unit used to send information to another unit that does not receive any information. A second type is Feed-back, the information can flow in multiple directions. Feed-back ANN allows Feed-back loops. The Back-propagation is used to calculate the error at the output unit. This error is back-propagated to all the units such that the error at each unit is proportional to its contribution towards the total error. The errors at each unit are used to optimize the weight at each connection. We can use linear regression as a more efficient method of training the model. If we add an intermediate layer with hidden neurons, the ANN becomes non-linear. This is known as a multilayer Feed-Forward ANN, where each layer of nodes receives inputs from the previous layers. The outputs of the nodes in one layer are inputs to the next layer. The inputs to each node are combined using a weighted linear combination. The result is modified by a nonlinear function before being output.

When we train ANN, we divide dataset into three sets. Training set, Validation dataset and Test set. Training set is used to find the relationship between dependent and independent variables. The test set assesses the performance of model. The experimental performance of set of ANN models are estimated, compared, and ordered.. The commonly cross validation $(\mathrm{CV})$ technique is that the $k$-fold cross validation. This method can be referred as a resampling process. Every data point gets a chance to be in test set and training set, thus this method reduces the dependence on test-training split and reduces the variance of performance. The extreme case of $k$-fold $\mathrm{CV}$ will occur when $k$ is equal to number of data points.

We have evaluated our ANN model using the residual methods such as RMSE for the test set. [18-21]

\subsection{Packages of ANN and ARIMA Time Series Models}

There are many packages allow us to compute ANN models. We can apply the algorithm of ANN regression model using the "neuralnet" package after scaling the data, and splitting it to train and test sets. Train ANNs using the Back-propagation, Resilient Back-propagation with or without weight back-tracking, or using the globally version.

The ("forecast","nnet") packages are used for ANN time series forecasting using Feed-Forward ANN with a single hidden layer and lagged inputs for forecasting univariate time series. The "nnetar" function fits the model. A total of repeats networks are fitted. For non-seasonal data, the fitted model is denoted as an $\operatorname{NNAR}(p, 0, k)$ model, where $k$ is the number of hidden nodes. This is analogous to $\operatorname{AR}(p)$ model but with non-linear functions. The default is the optimal number of lags according to the AIC for linear $\operatorname{AR}(p)$ model. For seasonal data, the fitted model is called an $\operatorname{NNAR}(p, P, k)[m]$ model, which is analogous to an $\operatorname{ARIMA}(p, 0,0)(P, 0,0)[\mathrm{m}]$ model but with nonlinear functions.

With seasonal data, we add the last observed values from the same season as inputs. For example, $\operatorname{NNAR}(3,1,2)$ [12] model has inputs, $\left[y_{t-1}, y_{t-2}, y_{t-3}\right], \ldots ., y_{t-12}$, and two neurons in the hidden layer. $\operatorname{NNAR}(p, P, 0)[m]$ model is equivalent to an $\operatorname{ARIMA}(p, 0,0)(P, 0,0)[m]$ model without the restrictions on the parameters that ensure stationarity. [22-26]

\subsection{VARS Models}

The vector autoregressive models (VARS) are used for multivariate time series. Each variable is a linear function of the past lags of itself and the past lags of other variables. Suppose that three different time series variables, denoted by $x_{(t, 1)}, x_{(t, 2)}$ and $x_{(t, 3)}$. VAR of order 1 is denoted by $\operatorname{VAR}(1)$, can be presented as:

$$
\begin{aligned}
& x_{(t, 1)}=\alpha_{1}+\phi_{11} x_{t-1,1}+\phi_{12} x_{t-1,2}+\phi_{13} x_{t-1,3}+w_{t, 1} \\
& x_{(t, 2)}=\alpha_{2}+\phi_{21} x_{t-1,1}+\phi_{22} x_{t-1,2}+\phi_{23} x_{t-1,3}+w_{t, 2} \\
& x_{(t, 3)}=\alpha_{3}+\phi_{31} x_{t-1,1}+\phi_{32} x_{t-1,2}+\phi_{33} x_{t-1,3}+w_{t, 3}
\end{aligned}
$$


Each variable is a linear function of the lag 1 values for all variables in the set. In a VAR(2) model, the lag 2 values for all variables are added to the right sides of the equations, In the case of three variables (time series), there would be 6 predictors on the right side of each equation: 3 lags 1 terms and 3 lags 2 terms. In general, for $\operatorname{VAR}(p)$ model, the first $p$ lags of each variable in the system would be used as regression predictors for each variable. VAR models are a specific case of more general VARMA models. VARMA models for multivariate time series include the VAR structure above along with moving average terms for each variable. These are special cases of ARMAX models that allow for the addition of other predictors that are outside the multivariate dataset. Here, we will fit the model of the form:

$$
x_{t}=\Gamma u_{t}+\phi x_{t-1}+w_{t}
$$

where $u_{t}=(1, t)^{\prime}$ includes terms to simultaneously fit the constant and trend.

Granger causality test is a statistical test for determining whether one time series is useful in forecasting another time series. A time series $X$ is said to Granger-cause $\mathrm{Y}$ if it can be shown that those $X$ values provide statistically significant information about future values of $\mathrm{Y}$ (using $\mathrm{F}$ test). Multivariate Granger causality analysis is performed by VAR to the time series. Let $x(t) \in \mathbb{R}^{d \times 1}$ for $d$-dimensional multivariate time series. Granger-causality is performed by fitting a VAR model with $L$ time lags as follows:

$$
x(t)=\sum_{\tau=1}^{L} A_{\tau} X(t-\tau)+\varepsilon(t)
$$

Where $\varepsilon(t)$ is a Gaussian random vector, and $A_{\tau}$ is a matrix for every lag, for $\tau=1, \ldots, L$. Time series $X_{i}$ is called Granger cause of another time series, $X_{j}$, if at least one of elements $A_{\tau}(j, i)$ is significantly larger than zero. [27-28]

\subsection{Accurate Measures (AMs)}

There are some measures of model accuracy that reflects how much the predicted values close to the actual observed values. The measure ME refer to Mean Error, MAE refers to Mean Absolute Error, and presents information on long term performance of the models, the lower MAE the better is the long term model prediction. MAPE refers to Mean Absolute Percentage Error. MSE refers to Mean Squared Error. The differences between MSE and MAPE in determining the accuracy of a forecast. MSE is scale-dependent, MAPE is not. MAPE measures the accuracy across time series with different scales. MAPE is preferred because. MAPE also cannot be used when the time series take zero values. MASE refers to Mean Absolute Scaled Error, it is fraction between MAE of the forecast values, and MAE of the in-sample one-step naive forecast. RSE refers to Relative Squared Error, the lower RSE the better model prediction. MPE refers to Mean Percent Error, it is well known measure that corrects the results, and can be used to analyze different predictions. RMSE refers to Root of MSE, it is easily to interpret the model accuracy, the lower RMSE, the more accurate. Coefficient of determination $R^{2}$, it is used also for comparison between the ANN models. The $R^{2}$ measures the variance that is interpreted by the model. The problem of residual evaluation methods is that: it does not inform us about the model behavior when new data is introduced. We overcome this problem by splitting our data into training and test set. Constructing the model on training set, and evaluating the model by calculating RMSE for the test set. These measures are good measures of the overall predictive accuracy. [29-33]

The expressions of all measures are given below:

$$
\begin{aligned}
& \text { 1. MSE }=\frac{1}{n} \sum_{i=1}^{n}\left(\text { 染 }-y_{i}\right)^{2}, 2 . \mathrm{ME}=\frac{1}{n} \sum_{i=1}^{n}\left(y_{i}-y_{i}\right), 3 \cdot \mathrm{R}^{2}=1-\frac{\sum_{i=1}^{n}\left(\hat{y}_{i}-y_{i}\right)^{2}}{\sum_{i=1}^{n}\left(\operatorname{Mean}\left(\hat{y}_{i}\right)-y_{i}\right)^{2}} \\
& \text { 4. MAE }=\frac{1}{n} \sum_{i=1}^{n}\left|\hat{y}_{i}-y_{i}\right|, \quad \text { 5. MAPE }=\frac{1}{n} \sum_{i=1}^{n} \frac{y_{i}-\hat{y}_{i}}{y_{i}} \times 100,6 . \operatorname{RSE}=\frac{\sum_{i=1}^{n}\left(\hat{y}_{i}-y_{i}\right)^{2}}{\sum_{i=1}^{n}\left(\operatorname{Mean}\left(y_{i}\right)-y_{i}\right)^{2}} \\
& \text { 7. RMSE }=\sqrt{\frac{1}{n} \sum_{i=1}^{n}\left(y_{i}-\hat{y}_{i}\right)^{2}} \text {, where } y_{i} \text { and } \hat{y}_{i} \text { are observed and predicted values. }
\end{aligned}
$$

\section{Numeric Analysis}

ANNs are used to solve many artificial intelligence problems. They often better than the traditional machine learning models because they deal with non-linearity variable relations, and customization. In this section, first we specify the used dataset indicate its features and decompose the periodic time series. Then we apply the technique of ANN regression model using the "neuralnet" package after scaling (normalizing) the original data and test the ANN predictions. Then, we deal with the same dataset as a time series and apply 
the nnetar() function to fitting the multivariate time series and explain predictions accuracy. After that we decompose the seasonal data and applied the same function on the seasonal data and also explain the predictions accuracy. Finally, we apply the GLM on the multivariate time series and seasonal data and got some comparable results. In the other way we discuss the ARIMA analysis beginning with checking the stationary of time series and transform it to stationary time series using the difference way. Finally, we use the VARS modeling via determining the maximum lags according to many criteria and test the causality and instantly of the best ARIMA model, and forecast the VAR model.

\subsection{Dataset Features}

In this study, the selected data are explained the average monthly Exchange Rate values, and the related monthly transactions values for some sectors in the Egypt Stock
Exchange Market, during the period from January 2015 to February 2019. Therefore, we divided these data into 13 variables, the first one is an independent variable (represents the average monthly Exchange rate), and the remaining 12 variables (represent the monthly transactions values' sectors) that are dependent variables (not as a group). These sectors are: Communications, Financial services (excluding Banks), Real Estates, Tourism and Entertainment, Construction and Building, Household and Personal Products, Services Industrial Products and Cars, Food and Beverages, Banks, Healthcare and Medicines, Basic Resources, and Chemicals. The aim of this study is to know whether or not there is a statistically significant effect of the Exchange rate on the transactions values of the sectors in the Egypt Stock Exchange.

The plots of multivariate time series data can be displayed in Figure 2:

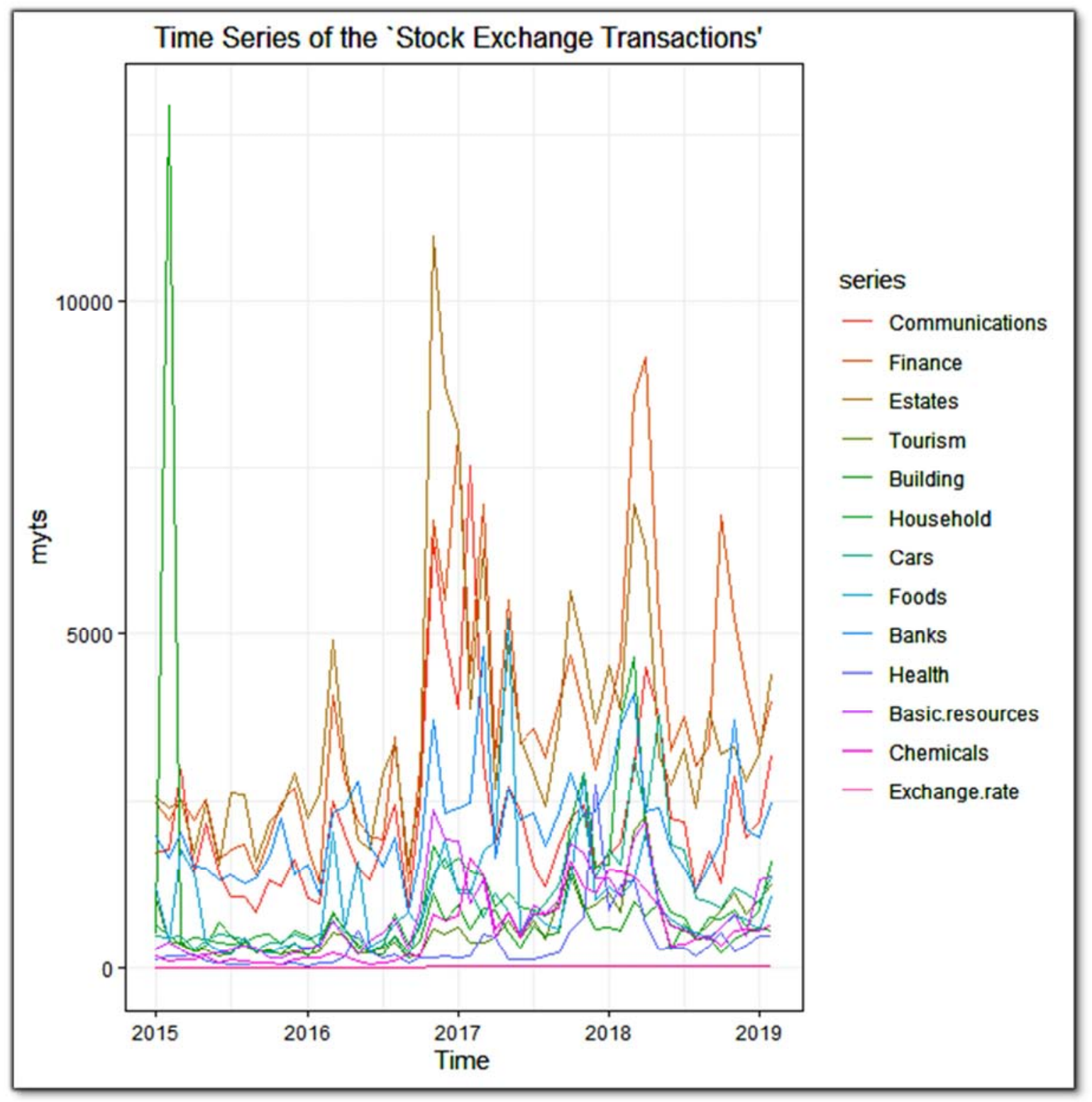

Figure 2. Multivariate time series plots. 
We can construct the seasonal, trend and remainder time series (for example; Exchange rate), in Figure 3.

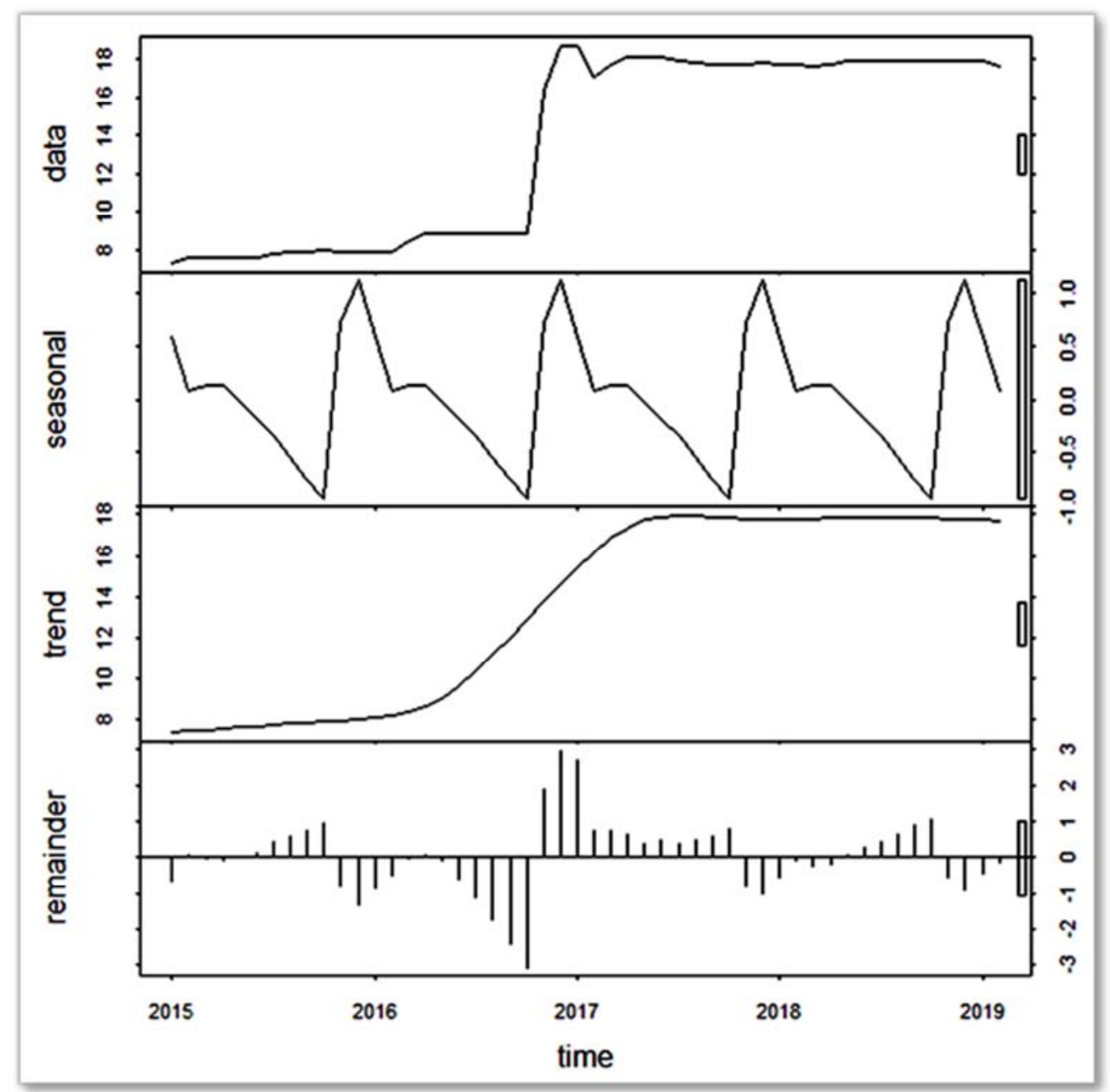

Figure 3. Seasonal, trend and periodic time series Exchange rate plots.

\subsection{ANN of Multivariate Non-time Series Data}

The "neuralnet" package, and the "neuralnet" function are used to construct the ANN and fit the multivariate non-time series data. Now we fit a ANN on our data. The first step is to scale the real dataset. Using unscaled may lead to meaningless results. The common techniques to scale data are: min-max normalization, Z-score normalization, median and MAD, and tan-h estimators. We scaled (normalized) all data to get reasonable results since error $=18264160$, and the reached threshold $=0.000948$. In addition, we discuss the results for the original data and the data as a seasonal data. We can split the data to $70 \%$ as the train data and $30 \%$ as test data. We used the "neuralnet" function to fit the model that the inputs represented by Exchange rate and the outputs the transactions' sectors.

Figure 4 displays the ANN fitted model for multivariate scaled data.

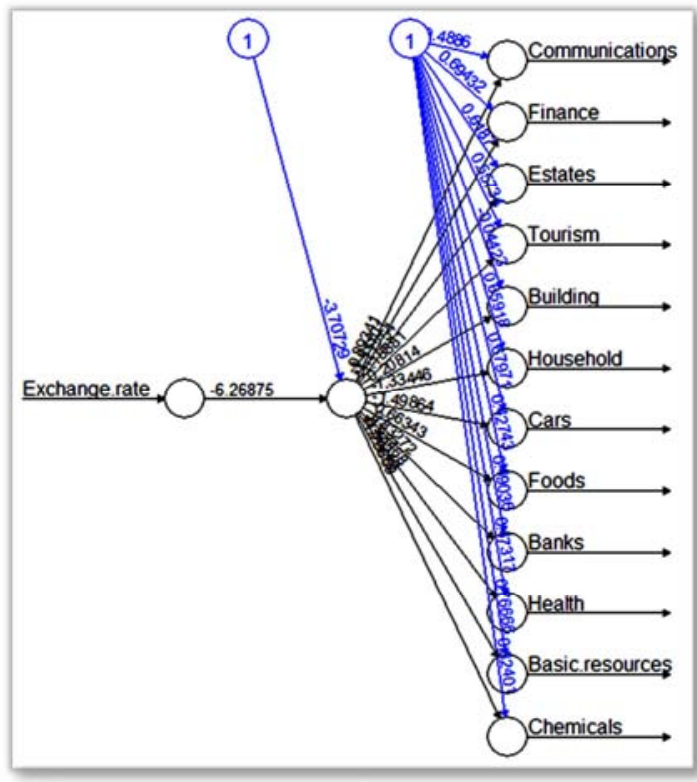

Figure 4. ANN fitting plot of multivariate non-time series data. 
Table 1 presents ANN fitted model:

Table 1. ANN fitted model.

\begin{tabular}{lll}
\hline Sectors & Intercept & Hidden layer \\
\hline Exchange rate & -3.707 & -6.269 \\
Communications & 0.489 & -0.893 \\
Finance & 0.694 & -1.411 \\
R. Estates & 0.619 & -1.173 \\
Tourism & 0.557 & -1.189 \\
Building & -0.044 & -0.208 \\
Household & 0.659 & -1.334 \\
Cars & 0.680 & -1.499 \\
Foods & 0.427 & -0.663 \\
Banks & 0.490 & -0.833 \\
Health & 0.473 & -0.935 \\
Basic resources & 0.767 & -1.502 \\
Chemicals & 0.824 & -1.797 \\
\hline
\end{tabular}

Error $=158.984$ Threshold $($ reached $)=0.0096$

Table 2 presents ANN first three predicted values for transactions' sectors:

Table 2. ANN first three predicted values for the sectors' variables.

\begin{tabular}{llll}
\hline Sectors & $\mathbf{1}$ & $\mathbf{2}$ & $\mathbf{3}$ \\
\hline Communications & -0.38789 & -0.38783 & -0.38782 \\
Finance & -0.68957 & -0.68947 & -0.68946 \\
R. Estates & -0.53095 & -0.53087 & -0.53086 \\
Tourism & -0.60827 & -0.60818 & -0.60817 \\
Building & -0.24839 & -0.24837 & -0.24837 \\
Household & -0.64944 & -0.64934 & -0.64933 \\
Cars & -0.79027 & -0.79016 & -0.79015 \\
\hline
\end{tabular}

\begin{tabular}{llll}
\hline Sectors & $\mathbf{1}$ & $\mathbf{2}$ & $\mathbf{3}$ \\
\hline Foods & -0.22296 & -0.22291 & -0.22290 \\
Banks & -0.32610 & -0.32604 & -0.32604 \\
Health & -0.44329 & -0.44322 & -0.44322 \\
Basic resources & -0.70672 & -0.70661 & -0.70659 \\
\hline
\end{tabular}

ANN model of non-time series data indicates that:

RMSE for Predicted values, using test set, for Dependent variables $=2.0279$.

$\mathrm{R}^{2}$ for Predicted values, using test set, for Dependent variables $=0.896$

RMSE for Predicted values, using test set, for Exchange rate as dependent variable $=0.1685$.

$\mathrm{R}^{2}$ for Predicted values, using test set, for Exchange rate as dependent variable $=0.987$.

With one hidden layer, the error $=158.984$ and reached threshold $=0.0096$.

Exchange rate forecasts: $\quad-1.1711 \quad-0.9314 \quad-0.7289$

These values reflects the model is accurate.

\subsection{ANN of Non-Seasonal Multivariate Time Series Data}

In this subsection, we used the "nnet" package to fit the time series data for all variables. We used the Exchange rate as a x-regressor time series. And used the "nnetar()" function for fitting all time series (12 variables as a time series) separately.

For accuracy model, we can present the next measures' values in Table 3:

Table 3. Accuracy values for each variable from training set (Non-seasonal data).

\begin{tabular}{|c|c|c|c|c|c|c|c|c|}
\hline Sector & X-reg. & ACF & ME & RMSE & MAE & MPE & MAPE & MASE \\
\hline Communications & Ex. rate & -0.0476 & 2.3451 & 224.334 & 157.669 & -3.1299 & 8.5984 & 0.11056 \\
\hline Finance & Ex. rate & 0.1214 & 0.1485 & 1122.744 & 873.023 & -7.561 & 21.7126 & 0.52823 \\
\hline R. Estates & Ex. rate & 0.0797 & 0.7949 & 1328.13 & 889.397 & -9.8352 & 23.0351 & 0.48841 \\
\hline Building & Ex. rate & 0.0006 & 0.1632 & 232.354 & 173.242 & -16.035 & 33.9895 & 0.28886 \\
\hline Household & Ex. rate & -0.0361 & -0.1948 & 218.709 & 147.582 & -9.8535 & 20.7944 & 0.20352 \\
\hline Cars & Ex. rate & -0.0308 & -0.1221 & 149.835 & 120.671 & -9.3211 & 18.655 & 0.18719 \\
\hline Banks & Ex. rate & -0.0765 & 0.0394 & 2.999 & 1.5661 & 0.00026 & 0.0675 & 0.00203 \\
\hline Health & Ex. rate & 0.1888 & 0.3479 & 151.9816 & 124.2541 & -51.611 & 72.823 & 0.33016 \\
\hline B. resources & Ex. rate & 0.0338 & -0.1550 & 337.6927 & 265.118 & -16.755 & 34.683 & 0.47259 \\
\hline Chemicals & Ex. rate & 0.0362 & 0.0362 & 4.0454 & 2.00349 & -0.18973 & 1.2265 & 0.00418 \\
\hline Exchange rate & X- reg. & -0.3545 & 0.00003 & 0.00289 & 0.00189 & 0.0001 & 0.0119 & 0.00056 \\
\hline
\end{tabular}

\subsection{ANN of Multivariate Seasonal Time Series Data}

Here we decompose the time series data to get the seasonal data. Accuracy values for seasonal data presented in Table 4:

Table 4. Accuracy values for each variable from training set (Seasonal data).

\begin{tabular}{|c|c|c|c|c|c|c|c|c|}
\hline Sector & X-reg. & $\mathrm{ACF}$ & ME & RMSE & MAE & MPE & MAPE & MASE \\
\hline Communications & Ex. rate & -0.2249 & -0.0223 & 237.964 & 183.146 & -3.6289 & 10.1586 & 0.12843 \\
\hline Finance & Ex. rate & -0.0092 & 0.0533 & 1035.24 & 764.067 & -10.989 & 22.933 & 0.4623 \\
\hline R. Estates & Ex. rate & 0.0627 & 1.7822 & 1122.20 & 822.218 & -10.6387 & 24.538 & 0.4515 \\
\hline Tourism & Ex. rate & -0.0649 & 0.0263 & 248.291 & 184.628 & -17.0418 & 35.0971 & 0.502 \\
\hline Building & Ex. rate & -0.0878 & 0.1818 & 197.79 & 142.961 & -18.69 & 34.4997 & 0.2384 \\
\hline Household & Ex. rate & 0.0254 & 0.1542 & 194.428 & 136.742 & -11.8317 & 21.9256 & 0.1886 \\
\hline Cars & Ex. rate & -0.1207 & -0.2693 & 140.97 & 110.41 & -9.069 & 17.76 & 0.1713 \\
\hline Banks & Ex. rate & 0.0734 & 0.4845 & 6.557 & 3.309 & 0.0018 & 0.1587 & 0.004 \\
\hline Health & Ex. rate & -0.2548 & -0.057 & 204.65 & 140.506 & -37.548 & 56.74 & 0.373 \\
\hline
\end{tabular}




\begin{tabular}{lllllllll}
\hline Sector & X-reg. & ACF & ME & RMSE & MAE & MPE & MAPE & MASE \\
\hline B. resources & Ex. rate & 0.1144 & 0.0404 & 290.72 & 232.128 & -19.36 & 35.93 & 0.414 \\
Chemicals & Ex. rate & 0.2837 & -0.0008 & 9.945 & 4.7837 & -0.5022 & 1.53 & 0.00998 \\
Exchange rate & X- reg. & -0.3093 & -0.00001 & 0.00021 & 0.00015 & -0.00651 & 0.0357 & Inf \\
\hline
\end{tabular}

The AMs values of seasonal time series Exchange rate, reset time series are $\mathrm{x}$-regressors, are lower than the AMs values for non-seasonal time series Exchange rate. This indicates that the ANN model of seasonal time series is better.

\subsection{GLM of Non-Seasonal Time Exchange Rate}

Table 5 presents the results of GLM fitting for non-seasonal Exchange rate:

Table 5. Results of GLM fitting for non-seasonal time series Exchange rate"

\begin{tabular}{|c|c|c|c|c|}
\hline Exchange rate (GLM) & Estimate & Std. Error & t value & P-value \\
\hline Intercept & 8.523 & 1.484 & 5.742 & $0.000001 * * *$ \\
\hline Communications & -0.0007 & 0.0005 & -1.320 & 0.1949 \\
\hline Finance & 0.0012 & 0.0005 & 2.318 & $0.0261 *$ \\
\hline R. Estates & 0.0001 & 0.0007 & 0.123 & 0.9027 \\
\hline Tourism & -0.001 & 0.0018 & -0.559 & 0.5793 \\
\hline Building & -0.00018 & 0.0002 & -0.745 & 0.4608 \\
\hline Household & -0.0022 & 0.001 & -2.209 & $0.033^{*}$ \\
\hline Foods & 0.000005 & 0.00057 & 0.008 & 0.9935 \\
\hline Banks & -0.0007 & 0.0009 & -0.793 & 0.4329 \\
\hline Health & 0.00066 & 0.0013 & 0.493 & 0.6247 \\
\hline Basic resources & 0.0006 & 0.0023 & 0.260 & 0.7963 \\
\hline Chemicals & 0.0069 & 0.00186 & 3.715 & $0.0007 * * *$ \\
\hline
\end{tabular}

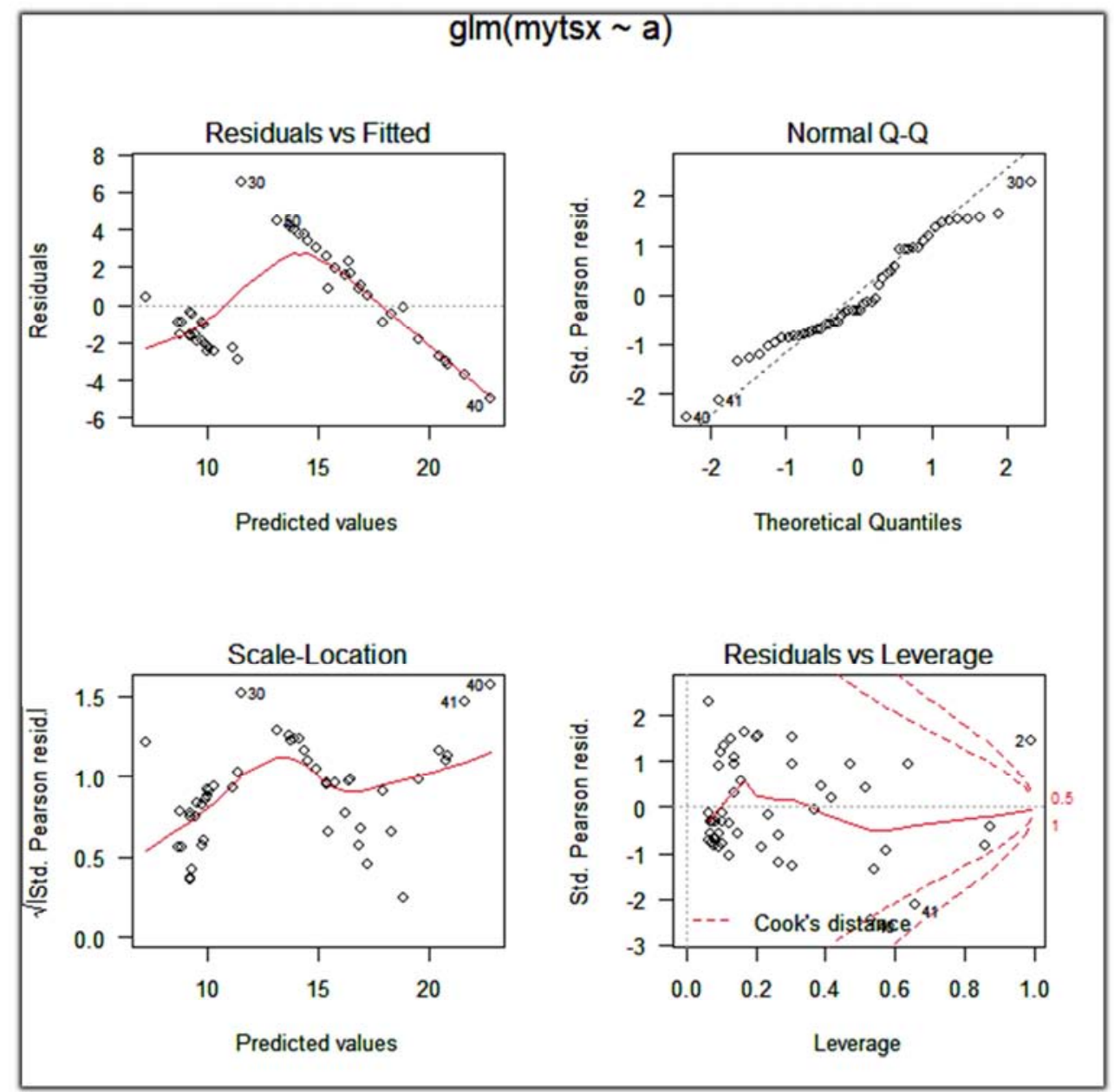

Figure 5. GLM fitting of non-seasonal time series Exchange rate.

Residual deviance: $\quad 325.51 \quad$ AIC: 263.56 


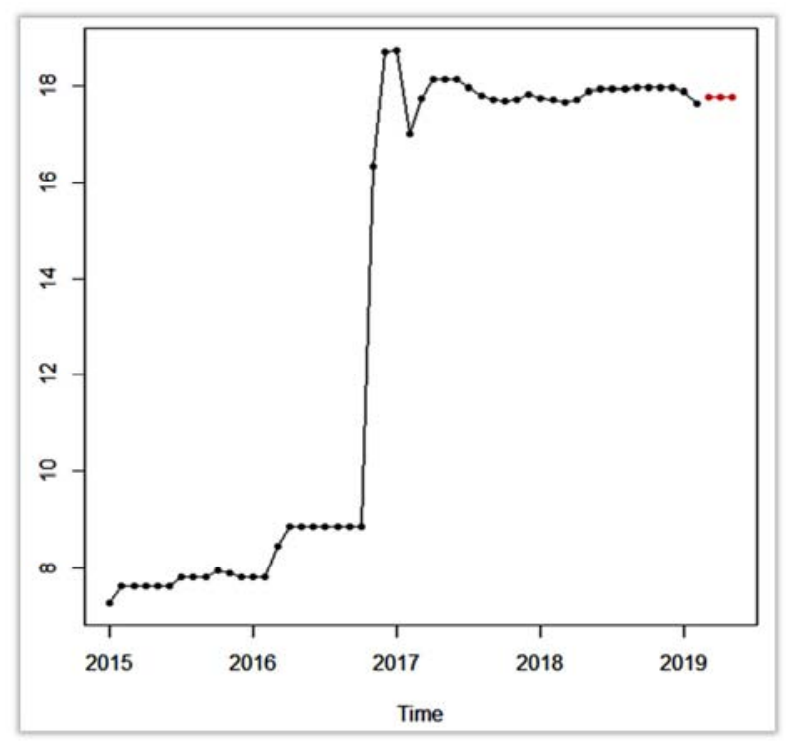

Figure 6. Predictions for 3 non-seasonal time series Exchange rate.

Exchange rate non-seasonal forecasts: $\quad 17.777 \quad 17.778 \quad 17.778$

\subsection{GLM of Seasonal Time Series Exchange rate}

Table 6 presents the results of GLM fitting for seasonal time series Exchange rate:

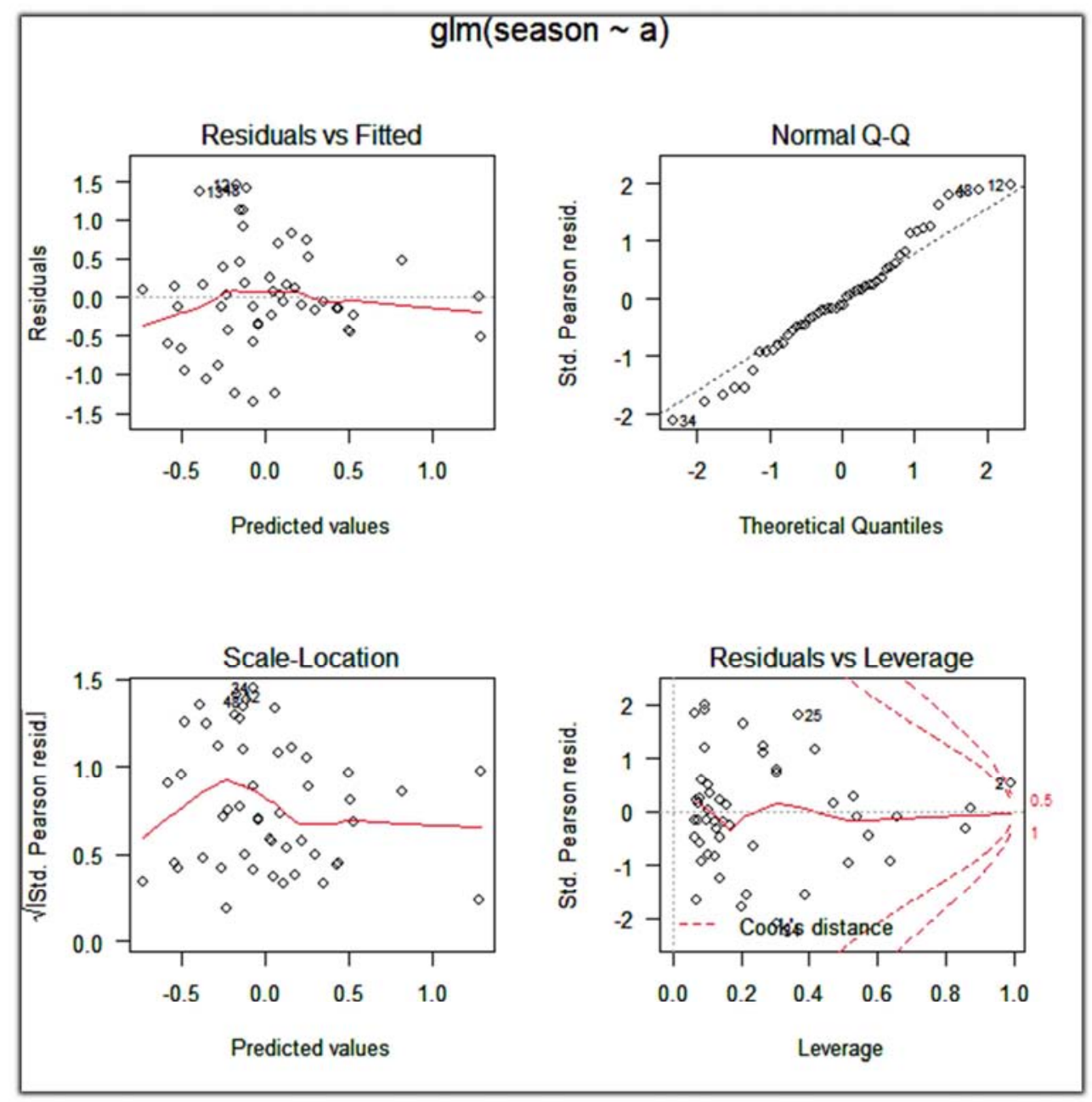

Figure 7. GLM fitting for seasonal time series Exchange rate. 
Table 6. Results of GLM fitting for seasonal Exchange rate.

\begin{tabular}{lllll}
\hline Exchange rate (GLM) & Estimate & Std. Error & t value & P-value \\
\hline Intercept & -1.039 & 0.3 .891 & -2.671 & $0.0112 *$ \\
Communications & 0.00017 & 0.00013 & 1.288 & 0.2057 \\
Finance & -0.00016 & 0.0001 & -1.230 & 0.2265 \\
R. Estates & 0.000236 & 0.00017 & 1.338 & 0.1890 \\
Tourism & 0.0003 & 0.00048 & 0.624 & 0.5362 \\
Building & 0.00001 & 0.00006 & 0.220 & 0.8268 \\
Household & -0.00016 & 0.00026 & -0.605 & 0.5491 \\
Cars & 0.00012 & 0.00026 & 0.466 & 0.6443 \\
Foods & 0.0001 & 0.00015 & 0.924 & 0.3614 \\
Banks & 0.00024 & 0.0002 & 1.006 & 0.3210 \\
Health & 0.0007 & 0.00035 & 1.947 & 0.0592 \\
Basic resources & -0.0005 & 0.0006 & -0.797 & 0.4304 \\
Chemicals & -0.0004 & 0.0005 & -0.814 & 0.4211 \\
\hline
\end{tabular}

Figure 7 displays the GLM fitting for seasonal time series Exchange rate:

Residual deviance: $\quad 22.372 \quad$ AIC: 129.68

Comparing between the residual deviance and AIC for the seasonal and non- seasonal time series Exchange rate forecasting, we found that it is lower for seasonal data. This indicate that GLM model is better for seasonal time series Exchange rate. However, the parameter estimates of Finance, Household and Chemicals sectors are significant for Exchange rate in non-seasonal time series case. Both of them contains the significance intercept of the model. Figure 8 displays the first three forecasts values for seasonal time series Exchange rate:

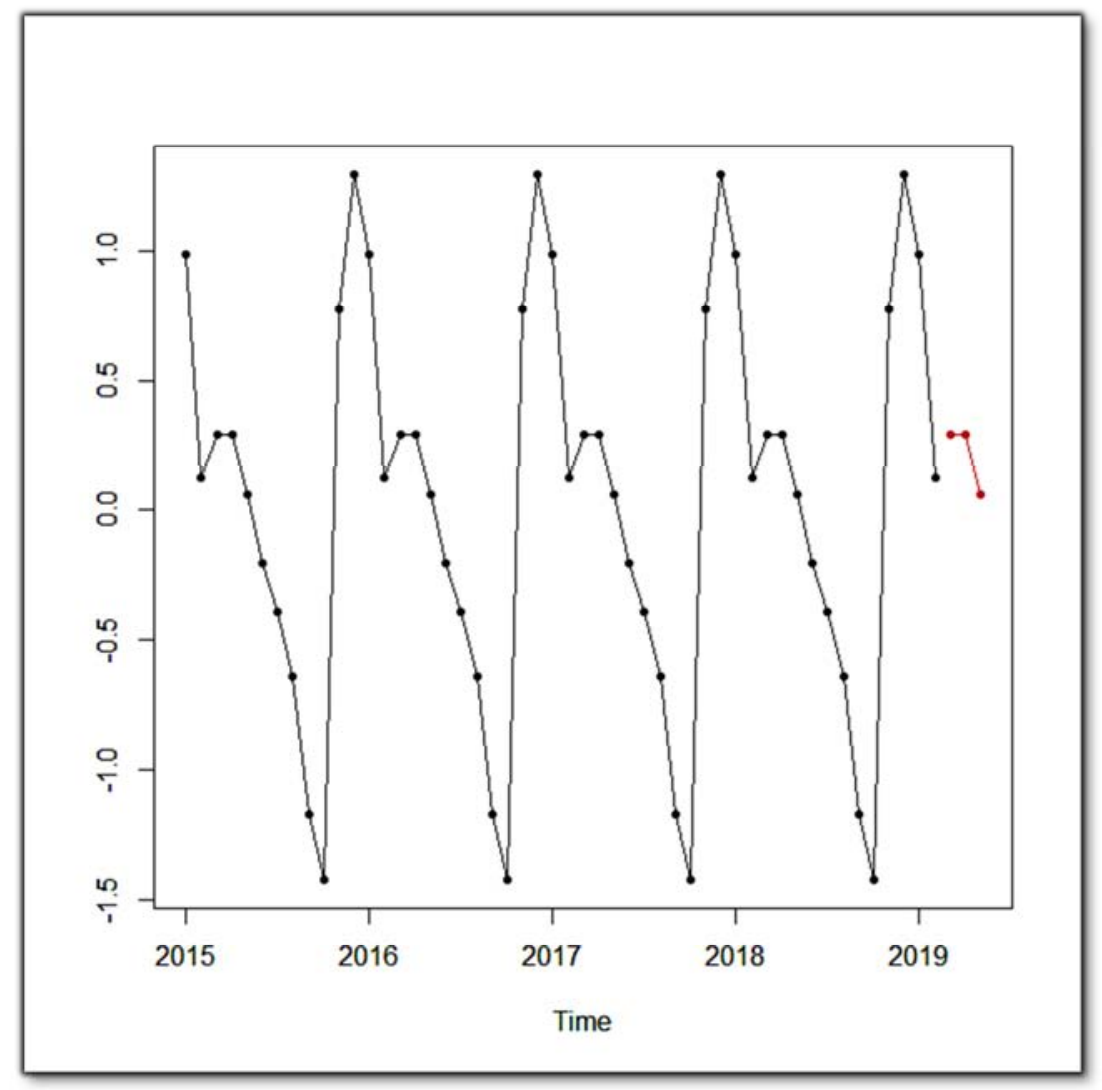

Figure 8. Forecasts of 3 seasonal time series data.

Exchange rate seasonal forecasts $\quad 0.29099 \quad 0.29038 \quad 0.0639$

\subsection{ARIMA of Multivariate Time Series Data}

For the time series data, we can use Adjusted Dickey Fuller (ADF) test for all testing the stationarity of these data using significance level 5\%. The results can be presented in Table 7: 

Multivariate Time Series

Table 7. Case of time series before and after differencing process.

\begin{tabular}{|c|c|c|c|c|c|c|}
\hline Time series & Before & P. value & Before & After & P. value & After \\
\hline Exchange rate & -1.115 & 0.64 & Non-stationary & -5.339 & 0.01 & Stationary \\
\hline Communications & -3.832 & 0.01 & Stationary & -8.865 & 0.01 & Stationary \\
\hline Finance & -3.573 & 0.011 & Stationary & -9.509 & 0.01 & Stationary \\
\hline R. Estates & -3.876 & 0.01 & Stationary & -8.783 & 0.01 & Stationary \\
\hline Tourism & -2.77 & 0.074 & Non-stationary & -7.544 & 0.01 & Stationary \\
\hline Building & -7.051 & 0.01 & Stationary & -19.88 & 0.01 & Stationary \\
\hline Household & -2.814 & 0.068 & Non-stationary & -6.31 & 0.01 & Stationary \\
\hline Foods & -5.497 & 0.01 & Stationary & -11.286 & 0.01 & Stationary \\
\hline Banks & -4.917 & 0.01 & Stationary & -10.568 & 0.01 & Stationary \\
\hline Health & -3.79 & 0.01 & Stationary & -10.699 & 0.01 & Stationary \\
\hline Basic Resources & -2.805 & 0.069 & Non-stationary & -7.966 & 0.01 & Stationary \\
\hline Chemicals & -2.092 & 0.285 & Non-stationary & -7.913 & 0.01 & Stationary \\
\hline
\end{tabular}

The ADF test indicates that the multivariate time series are non-stationary under significance level 5\%. The time series should be stationary, so we differenced the whole time series to make it stationary. After differencing the time series, P-values are become 0.01 , then all time series are become stationary. Figure 9 displays the plots of stationary multivariate time series:

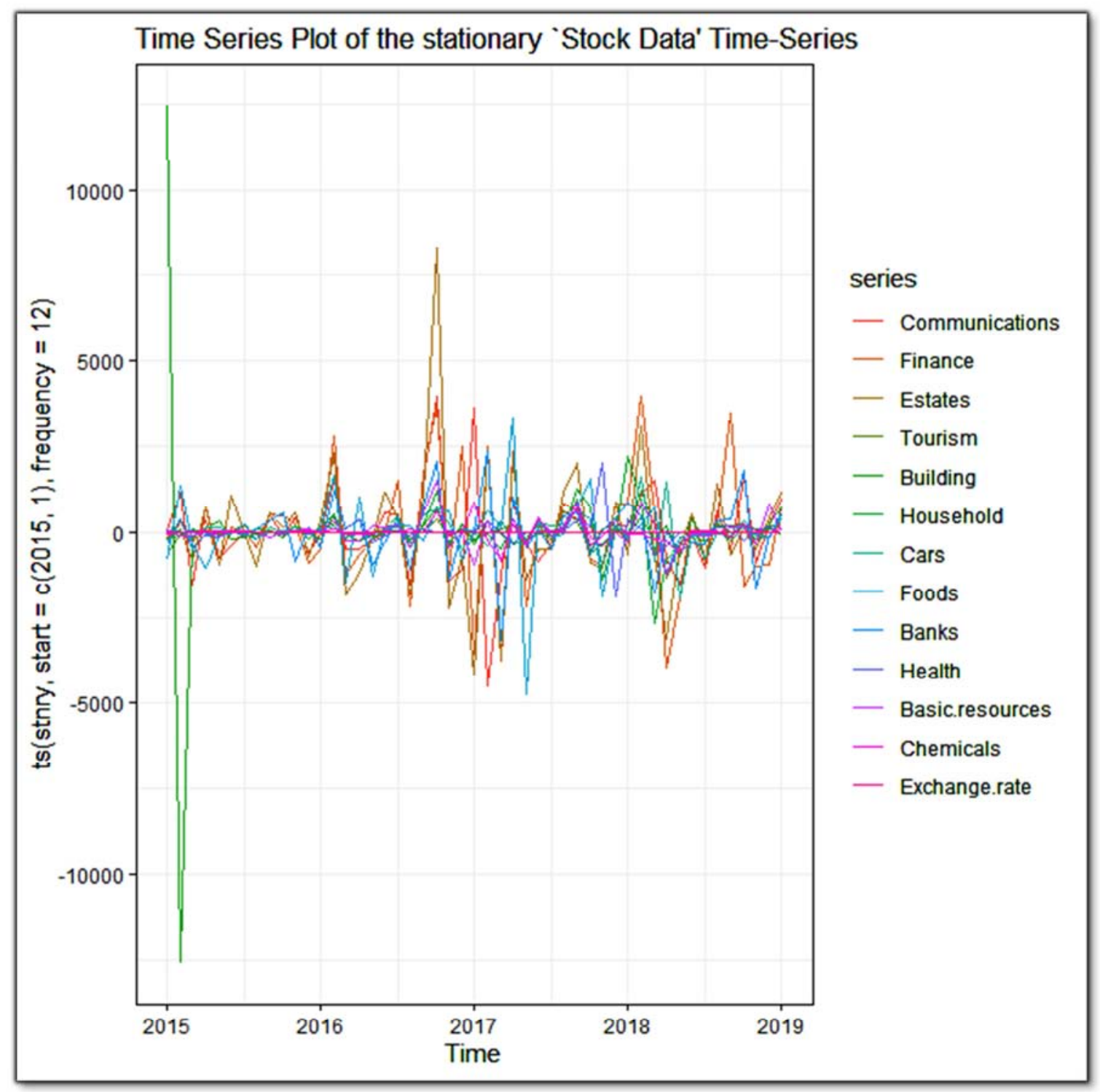

Figure 9. Stationary Multivariate time series plots.

Using the function "auto.arima()" for stationary time series, we got the models of ARIMA in Table 8: 
Table 8. ARIMA models.

\begin{tabular}{|c|c|c|c|c|c|}
\hline ARIMA model & Mean & Error & ARIMA Model & Mean & Error \\
\hline $\operatorname{ARIMA}(0,0,0)$ & zero mean & 155.609 & ARIMA(1,0,0) & zero & 154.464 \\
\hline $\operatorname{ARIMA}(0,0,0)$ & non-zero & 156.132 & $\operatorname{ARIMA}(1,0,0)$ & non-zero & 155.732 \\
\hline $\operatorname{ARIMA}(0,0,1)$ & zero mean & 154.054 & $\operatorname{ARIMA}(1,0,1)$ & zero & 156.293 \\
\hline $\operatorname{ARIMA}(0,0,1)$ & non-zero & 155.215 & $\operatorname{ARIMA}(1,0,1)$ & non-zero & 157.588 \\
\hline $\operatorname{ARIMA}(0,0,2)$ & zero & 156.228 & $\operatorname{ARIMA}(1,0,2)$ & zero & 157.268 \\
\hline $\operatorname{ARIMA}(0,0,2)$ & non-zero & 157.582 & $\operatorname{ARIMA}(1,0,2)$ & non-zero & Inf \\
\hline $\operatorname{ARIMA}(0,0,3)$ & zero & 155.793 & $\operatorname{ARIMA}(1,0,3)$ & zero & 158.174 \\
\hline $\operatorname{ARIMA}(0,0,4)$ & zero & 158.104 & $\operatorname{ARIMA}(1,0,4)$ & zero & 160.46 \\
\hline $\operatorname{RIMA}(0,0,4)$ & non-zero & 159.096 & $\operatorname{ARIMA}(1,0,4)$ & non-zero & 161.802 \\
\hline $\operatorname{ARIMA}(0,0,5)$ & zero & 160.394 & $\operatorname{ARIMA}(2,0,0)$ & zero & 155.723 \\
\hline $\operatorname{ARIMA}(2,0,1)$ & non-zero & 158.732 & $\operatorname{ARIMA}(3,0,1)$ & zero & 158.858 \\
\hline $\operatorname{ARIMA}(2,0,2)$ & zero & 157.990 & $\operatorname{ARIMA}(3,0,1)$ & non-zero & 159.734 \\
\hline $\operatorname{ARIMA}(2,0,2)$ & non-zero & 159.392 & $\operatorname{ARIMA}(3,0,2)$ & zero & 160.41 \\
\hline $\operatorname{ARIMA}(2,0,3)$ & zero & 160.4165 & $\operatorname{ARIMA}(3,0,2)$ & non-zero & 161.712 \\
\hline $\operatorname{ARIMA}(3,0,0)$ & non-zero & 158.075 & $\operatorname{ARIMA}(4,0,1)$ & zero & 160.841 \\
\hline $\operatorname{ARIMA}(4,0,1)$ & non-zero & 162.183 & $\operatorname{ARIMA}(5,0,0)$ & non-zero & 161.993 \\
\hline $\operatorname{ARIMA}(5,0,0)$ & zero mean & 160.797 & & & \\
\hline
\end{tabular}

From Table 8 . The Best model: $\operatorname{ARIMA}(0,0,1)$ with zero mean, with minimum error 154.054.

Exchange rate time series with the best model of $\operatorname{ARIMA}(0,0,1)$ with zero mean, we have the measures in Table 9:

Table 9. Measures of the best ARIMA $(0,0,1)$ model.

\begin{tabular}{lllllll}
\hline Coefficient & S.E. & Sigma2 & Log likelihood & AIC & AICc & BIC \\
\hline 0.2686 & 0.1243 & 1.269 & -74.9 & 153.79 & 154.05 & 157.58 \\
ME & RMSE & MAE & MPE & MAPE & MASE & ACF \\
0.1652106 & 1.1149 & 0.286 & NaN & Inf & 0.58 & -0.006 \\
\hline
\end{tabular}

All measures of accurate indicate that $\operatorname{ARIMA}(0,0,1)$ model is the best model, because all measures are achieved the lowest values.

To indicate the importance of stationary process for time series let us explain the plots for Exchange rate time series that effect of selection of the best model of ARIMA analysis.

Plot of non-stationary time series Exchange rate

Autocorrelation and Partial autocorrelation Functions, ACF and PACF respectively, to determine the order of models. Figure 10 displays the ACF and PACF for Non-stationary time series Exchange rate:

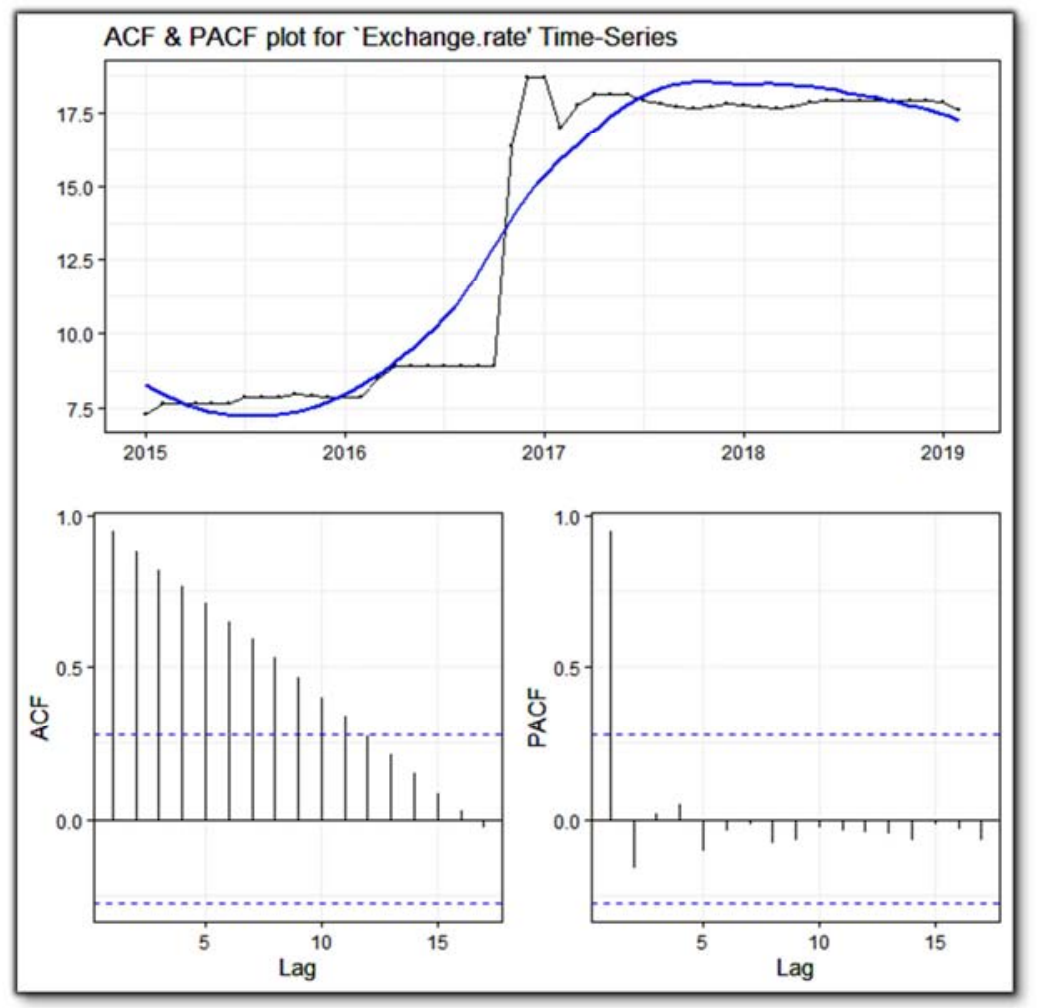

Figure 10. Non-stationary time series Exchange rate. 
Best ARIMA model $(0,1,1)$ with non-zero mean

Figure 11 displays ACF and PACF measures for Stationary time series Exchange rate

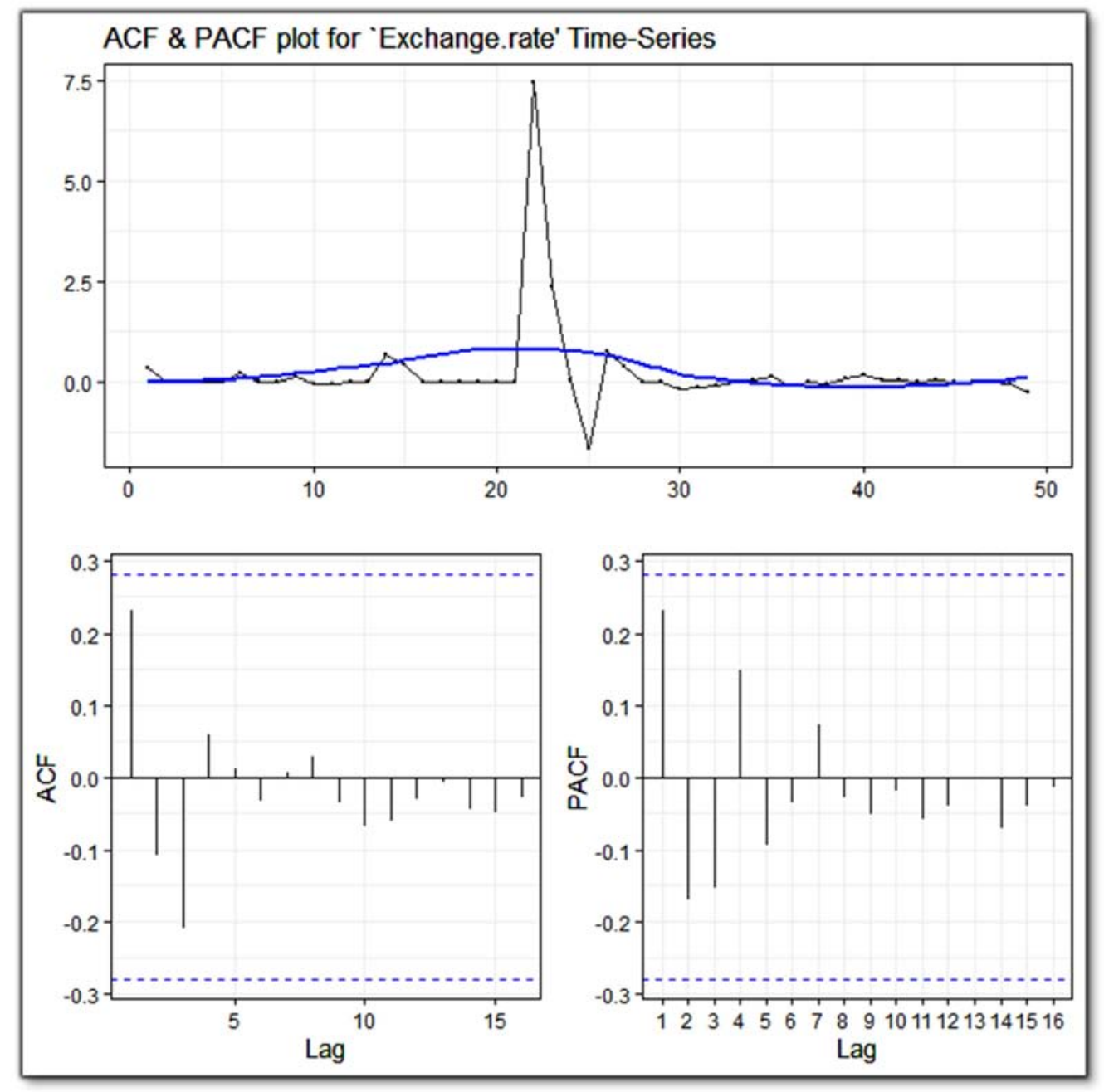

Figure 11. Stationary time series Exchange rate.

Best Arima Model $(0,0,1)$ with zero mean

Plots 10,11 of ACF and PACF measures for Exchange rate time series are explained that: the forecasting that based on stationary time series data, are more smooth and accurate.

\subsection{VARS of Multivariate Time Series Data}

We will use two different functions "VAR()", "vars::VAR()" to lag order identification, from two different packages in $\mathrm{R}$ "vars" and "MTS" respectively. Both functions are quite similar to each other but differ in their outputs. To identify the lag order for the VAR model, we obtained the estimations and its standard errors in Table 10:

Table 10. Results of VAR model.

\begin{tabular}{lll}
\hline ARIM(0,0,1)(GLM) & Estimates & Standard Error \\
\hline Exchange rate & 0.1707 & 0.1771 \\
Communications & 39.962 & 176.5 \\
Finance & 79.189 & 237.76 \\
R. Estates & 111.344 & 287.75 \\
Tourism & 24.0923 & 39.801 \\
\hline
\end{tabular}

\begin{tabular}{lll}
\hline ARIM(0,0,1)(GLM) & Estimates & Standard Error \\
\hline Building & -258.567 & 211.616 \\
Household & 26.5127 & 102.957 \\
Cars & 2.017 & 71.55004 \\
Foods & -19.47 & 141.6864 \\
Banks & 29.55 & 117.0024 \\
Health & 10.84 & 52.92681 \\
Basic Resources & 46.52 & 64.82822 \\
Chemicals & 1.655 & 37.3347 \\
AIC & 150.097 & \\
\hline
\end{tabular}

The "vars::VAR" function is a more powerful and convenient to identify the correct lag order, as shown for its results in Table 11:

Table 11. Results of VARS Model.

\begin{tabular}{lll}
\hline ARIM(0,0,1)(GLM) & Estimate & Std. Error \\
\hline Exchange rate & 0.4285 & 0.33950 \\
Communications & 0.00033 & 0.0002844 \\
Finance & -0.00002 & 0.0002242 \\
R. Estates & -0.0004 & 0.0004071 \\
Tourism & -0.0016 & 0.001531 \\
Building & -0.0001 & 0.0001591 \\
\hline
\end{tabular}




\begin{tabular}{lll}
\hline ARIM(0,0,1)(GLM) & Estimate & Std. Error \\
\hline Household & -0.0021 & 0.0006366 \\
Cars & 0.00163 & 0.0006193 \\
Foods & 0.00011 & 0.000245 \\
Banks & 0.0009 & 0.0004958 \\
Health & -0.0009 & 0.0007809 \\
Basic resources & 0.00206 & 0.001050 \\
Chemicals & 0.00196 & 0.001429 \\
AIC(n) & 14.65054 & \\
\hline
\end{tabular}

Comparing between the results of VAR and VARS models, we found that the model VARS is more accurate for ARIMA $(0,0,1)$ model. This is because the results of AIC and standard residuals of estimations for VARS model is lower than VAR model.

Granger Test for Causality:

For "causality" function to give reliable results, we need all the variables of the multivariate time series to be stationary. Cause variable here is Exchange rate. If not specified then first column of any regressor is used. Notice: Multiple variables can be used.

$\mathrm{H}_{0}$ : Exchange rate is not called a Granger cause of other time series.

$\mathrm{H}_{1}$ : Exchange rate is called a Granger cause of other time series.

F-test $=5.4419, \mathrm{P}$-value $=0.0000000000004$

Exchange rate is called a Granger cause of other time series. This means that it is useful in forecasting another time series with $5 \%$.

Instant Test:
$\mathrm{H}_{0}$ : No instantaneous causality between Exchange rate and other variables.

$\mathrm{H}_{1}$ : There is instantaneous causality between Exchange rate and other variables.

Chi-squared $=21.702, \mathrm{P}$-value $=0.041$

So, there is instantaneous causality between Exchange rate and other variables with $5 \%$.

We use a predict() function to forecast (over a short horizon) of VAR values, because the prediction (over long horizon) becomes unreliable or uniform. Table 12 presents the first three forecasting values for VARS:

Table 12. Forecasting VARS model.

\begin{tabular}{llll}
\hline Time series & $\mathbf{1}$ & $\mathbf{2}$ & $\mathbf{3}$ \\
\hline Exchange rate & -1.993 & -0.999 & -0.119 \\
Communications & -392.8023 & 678.211 & -457.121 \\
Finance & 637.586 & 193.227 & -14.832 \\
R. Estates & -806.122 & 531.746 & -205.7712 \\
Tourism & 408.768 & 4.8002 & -2.856 \\
Building & 185.742 & 190.747 & -295.9576 \\
Household & 340.975 & 971.142 & -81.5026 \\
Cars & -295.111 & 1006.891 & -231.859 \\
Foods & -1532.01 & 1172.727 & 239.588 \\
Banks & 595.628 & 561.261 & -223.907 \\
Health & 843.266 & -482.553 & 1097.624 \\
Basic resources & 229.308 & 330.11 & -145.795 \\
Chemicals & 524.843 & 308.633 & -347.984 \\
\hline
\end{tabular}

Figure 12 displays the forecast of all stationary time series:

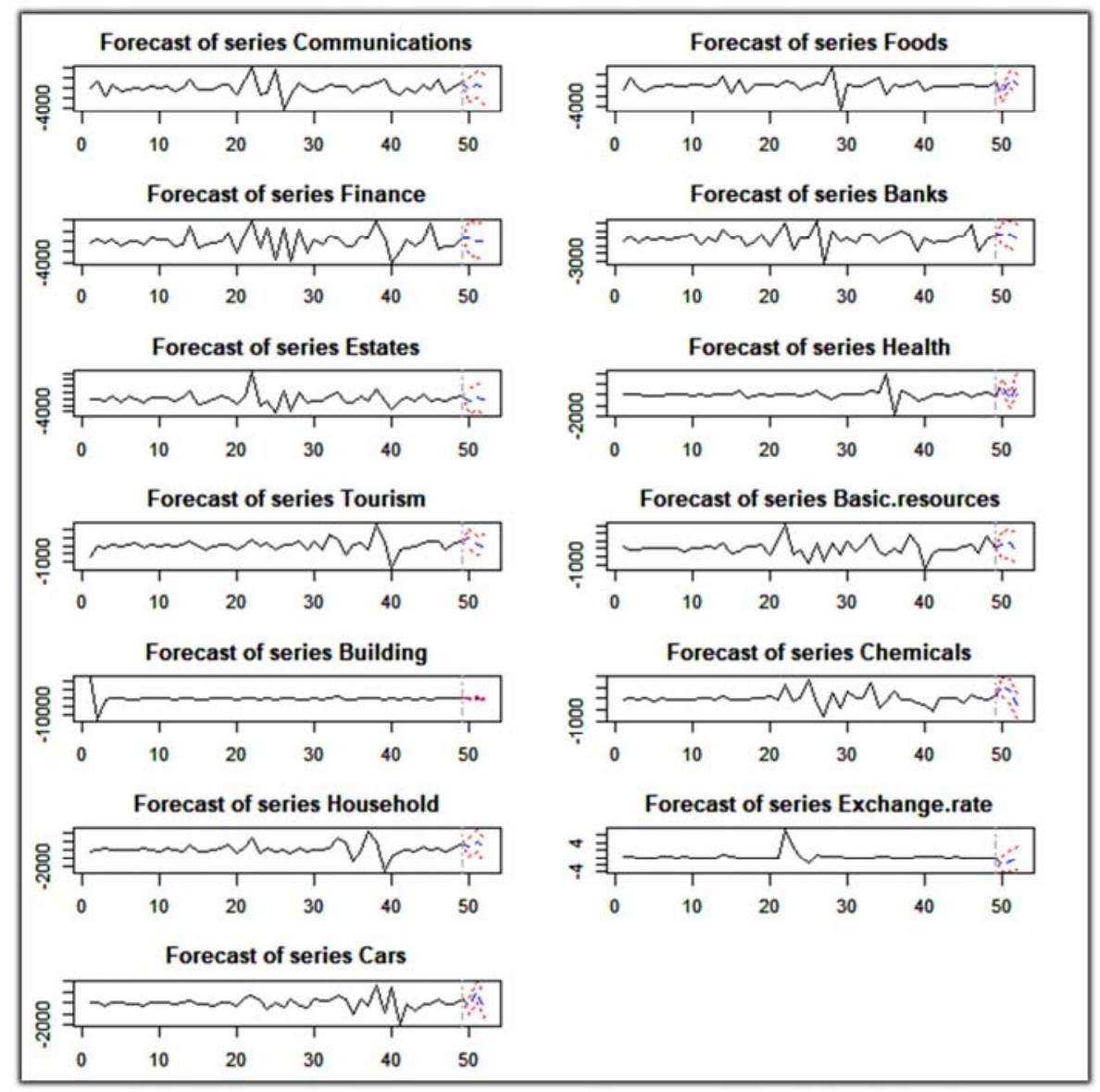

Figure 12. Forecasting the stationary time series. 


\section{Discussions}

From the previous results, we can summarize these results as below:

(1) We scaled (normalize) all non-time series data to get reasonable results since the error achieved a too high value $=$ 18264160 , and the reached threshold $=0.0009480014$.

(2) For ANN model of non-time multivariate series data, we found that:

RMSE for Predicted values, using test set, for Dependent variables $=2.0279$.

$\mathrm{R}^{2}$ for Predicted values, using test set, for Dependent variables $=0.896$.

RMSE for Predicted values, using test set, for Exchange rate as dependent variable $=0.1685$.

$\mathrm{R}^{2}$ for Predicted values, using test set, for Exchange rate as dependent variable $=0.987$.

With one hidden layer, the error $=158.984$ and reached threshold $=0.0096$.

All these values indicate the ANN model accuracy.

(3) For using the ANN model for forecasting the time series Exchange rate, we found:

The AMs values of seasonal time series Exchange rate, reset time series are X-regressors, are lower than the AMs values for non-seasonal time series Exchange rate. This indicates that the ANN model of seasonal time series is better.

(4) For using the GLM model for forecasting the time series Exchange rate, we found:

Comparing between the residual deviance and AIC for the seasonal and non- seasonal time series Exchange rate forecasting, we found that it is lower for seasonal data. This indicate that GLM model is better for seasonal time series Exchange rate. However, the parameter estimates of Finance, Household and Chemicals sectors are significant for Exchange rate in non-seasonal time series case. Both of them contains the significance intercept of the model.

(5) For using the ARIMA model for forecasting the multivariate time series data, we found:

As shown the ADF test indicates that the multivariate time series are non-stationary under significance level 5\%. The time series should be stationary, so we differenced the whole time series to make it stationary. After differencing the time series, P-values are become 0.01 , then all time series are become stationary. All measures of accurate indicate that $\operatorname{ARIMA}(0,0,1)$ model is the best model, because all measures are achieved the lowest values.

Figures 10, 11, that display ACF and PACF measures for Exchange rate time series are explained that the forecasting that based on stationary time series are more smooth and accurate.

(6) For using the VARS models for forecasting the multivariate time series data, we found:

Comparing between the results of VARS models, we found that the model VARS is more accurate for ARIMA $(0,0,1)$ model. This is because the results of AIC and standard residuals of estimations for VARS model is lower than VAR model.
Granger Test for Causality:

Exchange rate is called a Granger cause of other time series. This means that it is useful in forecasting another time series with $5 \%$.

Instant Test:

There is instantaneous causality between Exchange rate and other variables with $5 \%$.

\section{Conclusions}

This article discusses the theoretical and numerical aspects of different models that forecasting the multivariate time series data, whether these data are seasonal or not. These methods are ANN, ARIMA, GLM and VARS. All models are used to analyze the data that obtained from Egypt Stock Exchange for the Exchange rate, and the related transactions of twelve sectors. During the period from Jan 2015 to Feb 2019. We displayed the features of these data graphically, and also displayed the periodic features of these data. The ANN are to analyze these data in the Non-time series matter using the "neural" packages, after scaling these data to be reasonable and get good fitting results. We displayed the network of fitting process that considered the inputs is Exchange rate, and the related sectors' transactions are outputs indicating the weights of the net using one hidden layer. The ANN model is constructed, and the accuracy values of the model is constructed using two accurate measures RMSE and $\mathrm{R}^{2}$, and we have obtained the high accuracy in the case of considered the Exchange rate is input or output variable. Also, ANN is used to analyze the time series using "nnet" package using Exchange rate as a Xregressor (Exogenous variable). The process of forecasting and accuracy are investigated using ME, ACF, MAE, MPE, RMSE, MASE, and MAPE measures. This is done for Seasonal and non-seasonal time series data, and their results are compared. The GLM is used for Exchange rate is dependent variable, the model and estimations are tested and the predicted values for seasonal and non-seasonal time series data are explained numerically and graphically. ARIMA models are also used to analyze the multivariate time series data. Where we check the stationary of time series using ADF test. This test indicated that the most of time series data non-stationary. The differencing method, to make the time series to be stationary, is used. We retest the differenced multivariate time series data and found them are stationary (P-value $=0.01)$. More of ARIMA models are constructed, and selected the best model $\operatorname{ARIMA}(0,0,1)$ with zero mean, and minimum error, for Exchange rate time series, using auto.arima () function. The ACF and PACF plots are displayed before and after stationary process, for Exchange rate time series. The ARIMA $(0,0,1)$ model is tested using the measures AIC, BIC, AICc, MASE, ACF, MAPE, RMSE, MAE and ME. Finally, The VARS models are used to analysis the multivariate time series. The lag order is identified, the estimation of parameters are calculated, the standard errors for estimators are calculated, the measures of accuracy are indicated. Granger test for causality, with causality() function, 
indicated that Exchange rate is useful in forecasting other time series with 5\%. Also Instant test indicated that there is instantaneous causality between Exchange rate and other variables with significance level 5\%. The predicted values for Exchange rate are forecasted using the predict() function, and we explained that graphically. We indicate that the NNAR() model for fitting the ANNs for time series models is equivalent to an ARIMA() model, but without the restrictions on the parameters to ensure stationarity. Also, for seasonal data $\operatorname{NNAR}(p, P, 0)[m]$ model is equivalent to an $\operatorname{ARIMA}(p, 0,0)(P, 0,0)[m]$ model but without the restrictions on the parameters that ensure stationarity. For these data, we conduct that The ANN and GLM models of fitting seasonal time series is better than non-seasonal time series. The transactions of Finance, Household and Chemicals sectors are significant for Exchange rate in non-seasonal time series case. The forecasting that based on stationary time series data are more smooth and accurate. The VARS model is more accurate rather than VAR model for ARIMA $(0,0,1)$. Finally, we used the predict() function to forecast of VAR values over a short horizon, because the prediction over long horizon becomes unreliable or uniform.

\section{Acknowledgements}

To Al-Obour High Institute for Management \& Informatics. Department of Basic Science.

\section{References}

[1] Intrator, O. and Intrator, N. (1993). Using neural nets for interpretation of nonlinear models. Proceedings of the Statistical Computing Section, pages 244-249. San Francisco: American Statistical Society (eds).

[2] Zhang, G. et al. (1998). Forecasting with artificial neural networks: The State of the Art. International Journal of Forecasting, 14, pages 35-62.

[3] Al-Shawadfi, G. (2003). A comparison between neural network and Box-Jenkins Forecasting techniques with application to real data. King Saud University, King Fahd National Library Cataloging-in-Publication Data.

[4] Hothorn, et al. (2005). The design and analysis of benchmark experiments. Journal of Computational and Graphical Statistics, 14 (3), pages 675-699. doi/abs/10.1198/106186005X59630.

[5] LiHongHu, GuanHuaChen, G and Raymond Ming-WahChau (2006). A neural networks-based drug discovery approach and its application for designing aldose reductase inhibitors. Journal of Molecular Graphics and Modelling, 24 (4), pages 244-253. https://doi.org/10.1016/j.jmgm.2005.09.002

[6] Zou, C. and Zhou, L. (2007). QSAR study of oxazolidinone antibacterial agents using artificial neural networks. Molecular Simulation, 33 (6), pages 517-530. doi/abs/10.1080/08927020601188528.

[7] Eugster M., Hothorn, T. and Leisch, F. (2008). Exploratory and inferential analysis of benchmark experiments. Technical Report Number 030, Dept. of Statistics, University of Munich. https://epub.ub.uni-muenchen.de/4134/1/tr030.pdf
[8] Kose, E. (2008). Modelling of color perception of different age groups using artificial neural networks. Expert Systems with Applications, vol. 34, no. 3, pages 2129-2139. doi.org/10.1016/j.eswa.2007.02.036.

[9] Alshawadfi, G. and Hagag, A. (2013). Artificial intelligence and time series analysis. The Scientific Journal of the Faculties of Commerce Sector, Al-Azhar University 10, pages 572-612.

[10] Rostampour, V. et al. (2013). Using artificial neural network (ANN) technique for prediction of apple bruise damage. Austrial Journal of Crop Secience, 7 (10), pages 1442-1448. www.cropj.com/rostampour_7_10_2013_1442_1448.pdf

[11] Doreswamy and Chanabasayya (2013). Performance analysis of neural network models for Oxazolines and Oxazoles derivatives descriptor dataset. International Journal of Information Sciences and Techniques, 13 (6). DOI: 10.5121/ijist.2013.3601.

[12] Hanjouri, M. and Abu Qamar, A. (2018). A comparative study of the ANN and ARIMA models for predicting global sugar prices. Al-Azhar University Journal, Gaza, Public Human Series, 20, special issue A, pages 212-230.

[13] Mills, C. (1990). Time series techniques for economists. Cambridge University Press.

[14] Box, P. and Jenkins, M., and Reinsel, C. (1994) Time Series Analysis Forecasting and Control, 3rd edition. Prentice -Hall Inc., New Jersey. http://www.sciepub.com/reference/40199

[15] Englewood Cliffs, NJ: Prentice-Hal 1, page 151.

[16] Asteriou, D. and Hall, G. (2011). ARIMA models and the BoxJenkins methodology. Applied Econometrics $\left(2^{\text {nd }}\right.$ ed). Palgrave MacMillan. pages 265-286. doi: 10.12691/jfe-3-1-4.

[17] Hyndman, R. and Athanasopoulos, G. (2015). Seasonal ARIMA models. Forecasting: principles and practice.

[18] Swain, S et al. (2018). Development of an ARIMA model for monthly rainfall forecasting over Khordha District, Odisha, India. Advances in Intelligent Systems and Computing, 708, pages 325-331. doi.org/10.1007/978-981-10-8636-6_34.

[19] Riedmiller, M. and Braun, H. (1993). A direct adaptive method for faster back-propagation learning: The RPROP algorithm. Proceedings of the IEEE International Conference on Neural Networks (ICNN), pages 586-591. San Francisco.

[20] Murata, N., Yoshizawa, S. and Amari, S. (1994). Network information criterion - determining the number of hidden units for an artificial neural network model. IEEE Transactions on Neural Networks, 5 (6), pages 865-872. DOI: 10.1109/72.329683.

[21] Riedmiller, M. (1994). Description and implementation details. Technical Report. University of Karlsruhe.

[22] Anastasiadis, A., Magoulas, G. and Vrahatis, M. (2005). New globally convergent training scheme based on the resilient propagation algorithm. Neuro computing 64, pages 253-270. doi.org/10.1016/j.neucom.2004.11.016.

[23] Hyndman, J. and Khandakar, Y. (2008). Automatic time series forecasting: Package "forecast" for R. Journal of Statistical Software, 27 (3), pages 1-23. doi: 10.18637/jss.v000.i00.

[24] Frauke, G. and Fritsch, S. (2010). Package "neuralnet": Training of Neural Networks. The R Journal, 2 (1), pages 30-38. https://journal.r-project.org/archive/2010-1/R 
[25] Fritsch, S. and Guenther, F. (2019). Package "neuralnet ": Training of Neural Networks: R package, version 1.44.2. cran.r-project.org/web/packages/neuralnet/neuralnet.pdf

[26] Hyndman, R. et al. (2020). Package "forecast": Forecasting functions for time series and linear models. R package, version 8.13. https://cloud.r-project.org/package=forecast

[27] Ripley, B. and William Venables, W, (2021). Package "nnet": Feed-Forward Neural Networks and Multinomial Log-Linear Models. package, version 7.3-15. https://cran.r-project.org/web/packages/nnet/nnet.pdf

[28] Granger, C. (1969). Investigating causal relations by econometric models and cross-spectral methods. Econometrica, 37 (3), pages 424-438.

[29] Lütkepohl, H. (2005). New introduction to multiple time series analysis (3rd ed.). Berlin: Springer. pages. 41-51. ISBN 978-3-540-27752-1.
[30] Makridakis, S. (1993). Accuracy measures: theoretical and practical concerns. International Journal of Forecasting. 9 (4), pages 527-529. DOI: 10.1016/0169-2070(93)90079-3.

[31] Diebold, X. and Mariano, R. (1995). Comparing predictive accuracy. Journal of Business and Economic Statistics, 13 (3), pages 253-265. doi/abs/10.1080/07350015.1995.10524599

[32] Hyndman, J. and Koehler B. (2006). Another look at measures of forecast accuracy. International Journal of Forecasting, 22 (4), pages 679-688. https://robjhyndman.com/publications/another-look

[33] Franses, Ph. H. B. F. (2015). A note on the Mean Absolute Scaled Error. International Journal of Forecasting, 32, pages 20-22. doi: 10.1016/j.ijforecast.2015.03.008. 